\title{
A Functional Approach to an Early Diagnosis and Early Intervention Program for Pre-School Children with Special Educational Needs in Hong Kong
}

\author{
Sun Tien-Lun Catherine, Zhou De-Hui Ruth, Kwok Sin-Hang Helen, \\ Yu Chun-Yeung Isaac, Wong Ka-Ying Shirley, Lo Man-Chung Sonia \\ Department of Counselling \& Psychology, Hong Kong Shue Yan University, Hong Kong, China \\ Email: tlsun@hksyu.edu,dhzhou@hksyu.edu,kwok_crc@hksyu.edu,cyyu_crc@hksyu.edu, \\ wong_crc@hksyu.edu, mclo_crc@hksyu.edu
}

Received October 2013

\begin{abstract}
This paper briefly introduces the framework of an early diagnosis and early intervention program for Hong Kong pre-school children with special educational needs launched by the Counselling \& Research Center at the Hong Kong Shue Yan University and Lok Sin Tong (a charitable organization established under Cap 151 of the Hong Kong Ordinances). The whole program involves 103 pre-school children with SEN and their parents. Prior to enrolling in this program, most of the children have been assessed to have autism, ADHD, or dyslexia. In this program, the Mullen Scales of Early Learning (Mullen, 1995) or Childhood Autism Rating Scale (Schopler, Reichler, DeVellis, \& Daly, 1980) were administered to the children by counselling psychologists to measure their cognitive and motor abilities. In addition, their parents were also invited to participate in the survey interview of Vineland Adaptive Behavior Scales (Sparrow, Cicchetti, \& Balla, 2008) and Child Behavior Checklist (Achenbach, 1991, 1992). Based on these measures and clinical observation, an Individualized Development Plan (IDP) has been designed for each child. A functional approach, rather than a diagnostic approach, has been applied in designing the IDPs, communicating them to the parents, and providing the children with relevant individual and group counselling and therapeutic services. Through illustration of this approach, we hope to stimulate further clinical and public discussion in the arenas of issues faced by children with SEN and their parents, as well as the perceived and projected advantages of early diagnosis and early intervention.
\end{abstract}

Keywords: Pre-School Children with SEN; Individualized Development Plan (IDP); Early Diagnosis; Early Intervention; Functional Approach

\section{Introduction}

Although Hong Kong government has been working tirelessly in promoting integrative education (IE) and individualized education plan (IEP) for children with special educational needs (SEN) in primary and middle schools (Hong Kong Education Bureau, 2008, 2013; Yuen \& Westwood, 2001), yet it is common knowledge that kindergarten children who suffer similar problems have not received sufficient support and resources. The brain plasticity is a significant feature in early childhood. A number of oversea studies have shown that early diagnosis and early intervention can help high-risk children control or even reduce the incidence rate of getting severe autism disorder, dyslexia and attention deficit/hyperactivity disorder (ADHD) (Landa, 2008; Rappaport, Ornoy, \& Tenenbaum, 1998; Victoria, Areti, \& Pavls et al., 2011).

In Hong Kong, a number of clinical and academic scholars have been engaged in studying dyslexia (McBride-Chang, Shu, Zhou, \& Wat, 2003; McBride-Chang, Lam, Lam, Doo, Wong, \& Chow, 2008), autism (Chung, Luk, \& Lee, 1990; Lam \& Rao, 1993), and ADHD (Leung, Lee, Hung et al., 2004). Despite these and other academic efforts, there has always been a pressing need for effective counselling and therapeutic services, which can enable children, especially pre-school children with SEN, to enjoy continuous educational and developmental op- portunities.

In 2012, the Counselling \& Research Center at the Hong Kong Shue Yan University and Lok Sin Tong (a charitable organization established under Cap 151 of the Hong Kong Ordinances) launched a two-year collaborative early diagnosis and early intervention program for 103 Hong Kong pre-school children with SEN.

Before the implementation of the program, parents were invited to join a focus-group interview, in which they shared four primary concerns about raising young children at risk of SEN.

First, the resources available for pre-school children at risk of SEN were very limited. In fact, resource would not be provided, unless the severity of the child's symptom severity could be graded under a government-sanctioned clinical assessment, the waiting time for which was usually at least half a year.

Second, even if parents received a clinical report after the child had been assessed, they were only informed about the category of disorder their child belonged to, but were not told what was happening to him/her, or how they as parents should go about seeking the necessary help for their child.

Third, if the child is at the right place at the right time, he/she might be provided training and/or support by governmentsponsored agencies. Unfortunately, the lack of coordination between the agencies often meant that services provided by them tended to be either repetitive or non-responsive to the 
developmental needs of the child. In other words, integrated effort directed at serving this particular age-group of children with SEN did not exist.

Fourth, parents emphasized that supporting their children at risk of SEN was a perennial struggle for which they sorely needed supervision and guidance. Otherwise, they were at a total loss as to what they needed to do for their children at achieving important developmental milestones.

With these four concerns in mind, we aimed at designing a program which would 1) focus on the special needs of each child; 2) assist parents to understand more fully their children's predicament; 3) establish a closer collaboration with parents; 4) guide parents to seek for resources which were uniquely suited to the needs of their children; and 5) centre on continuous developmental achievements in lieu of symptom control.

\section{Lok Sin Tong Program}

The Lok Sin Tong (LST) program aims at developing an Integrated Case Management System for preschoolers, who were confirmed or suspected to have special educational needs. Specifically, these were children diagnosed with or suspected to have the features of Autism Spectrum Disorder, Attention Deficit/Hyperactivity Disorder, Special Learning Difficulties, Intellectual Disabilities or Developmental Delay, and were deemed to be able to benefit from the program..

In Hong Kong, children entered the primary school system at the age of 6 . If children were confirmed or suspected with any kind of special educational needs before 6 years old, parents might apply for government placement in such facilities and services as the Early Education and Training Centre, Integrated Child Care Centre, Special Child Care Centre, Integrated Program for Mildly Disabled Children in kindergartens, and so on. Unfortunately, the waiting time of the service placement was very long, and parents were often overwhelmed with worry and frustration during the waiting period. Hence the current program also aimed at providing professional psychological and training support for families waiting for government services who could not afford the high fees charged by the private sector. Apart from providing counselling or training support for children, this program additionally provides parents with knowledge about the symptoms and developmental journey of SEN-related disorders, as well as various home-based training techniques for their children.

The present Integrated Case Management System had a two-fold purpose: First, a comprehensive Individualized Developmental Plan (IDP) supported by assessment data, clinical observations, and parents' interview data was developed for each child. Second, based on the IDP, an evidence-based plan of intervention was laid out for the child to receive the mostneeded services.

In order to enhance children's overall functioning and fulfill their developmental needs, the emphasis of this project was on establishing an Individualized Developmental Plan (IDP) instead of an Individualized Educational Plan (IEP). We believe that each child goes through changes in skills development during a predictable time line. Unfortunately, children with or suspected to have special educational needs were likely to experience tremendous hardship in reaching the developmental milestones by the expected timeline. Thus, "development" was a more crucial task than education for this group of children.

An even more practical consideration was that most children in this research program had not yet entered the local primary school system, thus it was premature to design individualized educational programmes for them. Therefore, the emphasis of this research on development rather than education is more logical, practicable and sustainable.

The preamble of this two-year research program was to design an ongoing developmental plan for each child based on the assessment results of the Mullen Scales of Early Learning (Mullen, 1995) and the Vineland Adaptive Behavior Scales (Sparrow, Cicchetti, \& Balla, 2008).

Subjects were recruited city-wide by self-referral and/or referral by agencies and schools. Since this research aimed at reaching out to families who could not afford the higher fees of the private sector, the family income was set at a designated amount by referencing the median family income statistics in Hong Kong.

In the actual recruitment exercise, Lok Sin Tong distributed program leaflets to the Social Welfare Department, early education services, local kindergartens and primary schools. A press conference was organized to enlist the help of the media to spread the news, and the research program was also promoted on a local radio station. Once the parents were accepted into the program, they were asked to complete an application form which included information on the basic developmental history of the children. Parents were invited to join the information seminar so that they could have a macro view of the research program and its scope of services. During the information seminar, parents were asked to fill in the Child Behavior Checklist (Achenbach, 1991, 1992), and appointments were made with them for the initial intake session with counselling psychologists.

Each child underwent the assessment phase, and the results were used in designing the IDP. Intervention provided by the psychologists adhered closely to the IDP. However, some modifications were made based on ongoing assessments and evaluations.

\section{Assessment}

Altogether 103 pre-school children including 77 boys and 26 girls joined this program. Among them, 72 were under 5 and 31 were above 5 years of age.

Parents and primary caregivers were given the Achenbach System of Empirically Based Assessment of Child Behavior Checklist to fill in.

Later, at a separate appointment, the Mullen Scales of Early Learning was administered to each child by a qualified counselling psychologist, followed by the completion of Vineland-II by the parents. During the Mullen test, the child's primary caregiver sat either in a separate room or in a chair behind the child. The child and the examiner sat at a child-size testing table. If the child left his/her seat during the assessment, he/she was encouraged to return to his/her seat. If this proved to be too difficult, some of the assessment was conducted on the floor until the child eventually returned to the table.

In suspected cases of Autism Spectrum Disorder, the children were further assessed by the Childhood Autism Rating Scale (Schopler, Reichler, DeVellis, \& Daly, 1980).

\section{Assessment Tools}

Achenbach System of Empirically Based Assessment-Preschool/Child Behavior Checklist (P/CBCL; Achenbach, 1991; 
Achenbach, 1992; Achenbach et al., 1987). Two different sets of questionnaires, Preschool Child Behavior Checklist and Child Behavior Checklist, were used in this program. PCBCL was used for children aged 1.5 to 5 , and CBCL was used for children aged 6 to 18. PCBCL contains 100 items describing behavior, while CBCL has 113 items. Each of the item is rated on a 3 -point scale ranging from $0=$ not true; $1=$ somewhat or sometimes true; and 2 = very true or often true. These ratings are then combined and analyzed to form different syndromes scales. PCBCL has seven syndromes scales: Emotionally Reactive, Anxious/Depressed, Somatic Complaints, Withdrawn, Sleep Problems, Attention Problems, and Aggressive Behavior; while CBCL includes eight syndromes including: Anxious/ Depressed, Withdrawn/Depressed, Somatic Complaints, RuleBreaking Behavior, Aggressive Behavior, Social Problems, Thought Problems, and Attention Problems. Apart from these syndromes, the ASEBA helps identify children's potential disorders according to the DSM-IV criteria. PCBCL is geared towards identifying preschooler children's Affective, Anxiety, Pervasive Developmental Problems, Attention Deficit/Hyperactivity, and Oppositional Defiant Problems; while CBCL can identify children's Affective, Anxiety, Somatic, Attention Deficit/Hyperactivity, Oppositional Defiant, Conduct, Sluggish Cognitive Tempo, Obsessive-Compulsive, and Post-traumatic Stress problems.

Translated Version of the Mullen Scales of Early Learning (Mullen, 1995). The Mullen Scales measure cognitive and motor ability of young children. It can be administered to infants and children up to 68 months of age. T-scores, percentile ranks, and age equivalents can be computed for the five scales separately (Gross Motor, Visual Reception, Fine Motor, Expressive Language, and Receptive Language). The median internal consistency split-half coefficients for the five Mullen scales ranged from .75 to .83, and .91 for the composite (Mullen, 1995).

Vineland Adaptive Behavior Scales-Second Edition (Vineland-II) (Sparrow et al., 2005, 2008). Vineland Adaptive Behavior Scales measure personal and social skills needed for everyday living. It can be administered to a parent or caregiver. T-scores, percentile ranks, and age equivalents can be computed for the five scales separately (Communication, Daily Living Skills, Socialization, Motor Skills, and Maladaptive Behavior Index). Each domain is further divided into subdomains (i.e. Receptive, Expressive and Written in the domain of Communication; Personal, Domestic and Community in the domain of Daily Living Skills; Interpersonal Relationships, Play and Leisure Time, and Coping Skills in the domain of Socialization; Fine and Gross in the domain of Motor Skills; and Internalizing and Externalizing in Maladaptive Behavior Index). The Vineland Scales have good overall test-retest reliability and internal consistency on subdomains (Sparrow et al., 2005). In this research, Maladaptive Behavior Index was not analyzed as there are considerable overlaps with the scales in CBCL.

Childhood Autism Rating Scale (CARS; Schopler, Reichler, \& Rochen Renner, 1988). CARS is a parent-report behavioral rating scale used for assessing the presence and severity of symptoms of ASD among children of all ages. It contains 15 items on a four-point Likert scale (age-appropriate $=1$, mildly abnormal $=2$, moderately abnormal $=3$, severely abnormal $=$ 4). This scale assesses the severity of children's autistic disorder, with the total scores ranging from a low of 15 to a high of 60 . The clinical cut-off point of this scale is 30 , with scores below
30 indicating that the individual is on the non-autistic range, scores between 30 and 36.5 indicating a mild to moderate level of autism, and scores from 37 to 60 indicating a severe level of autism (Schopler et al., 1988). In the study of Chlebowski, Green, Barton and Fein (2010), their results revealed that CARS has high criterion-related validity, inter-rater and testretest reliability, and internal consistency.

\section{Assessment Reports}

A functional approach rather than a diagnostic approach was used to brief parents on the assessment results of their children. In this approach, counselling psychologists translated clinical terminology into language easily comprehended by parents. The psychologists did not simply report on children's weaknesses, but also emphasized strengths in developmental domains whenever appropriate. Alternatively stated, the functional approach does not focus on the diagnosis of abnormal symptoms, but rather on informing parents about their children's current status in the various developmental domains. The individual assessment report serves mainly as an evidence-based reference on which every child's IDP could be designed and implemented.

The descriptive statistical analyses of the data sets of these 103 children also allowed researchers to gain a holistic picture of their developmental status. These analyses were instrumental for the planning and administration of group-based interventions.

As shown in Table 1, the average scores of total problem CBCL for children under the age of 5 reached clinical significance $($ Mean $=68.00$, cut-off score $=64)$. Among all domains of the Child Behavior Checklist, children under the age of 5 showed withdrawn behaviors (Mean $=75.46$; cut off score $=70$ ) and internalizing problem (Mean $=67.88$, cut off score $=64$ ).

As shown in Table 2, the total problematic behaviors of children over the age of 6 also reached clinical significance (Mean $=67.56$, cut off score $=64)$. However, children above 6 years old obviously showed risky behaviors in different domains from those of children under 5 . In the current research, children over 6 showed attention problems $($ Mean $=71.78$, cut off score $=70$ ) and externalizing problem (Mean $=64.33$, cut off score $=64$ ). In addition, children over 6 also showed a risky tendency in aggressive behaviors, social problems, and thought problems, as their average scores all fell within the risky range of $65-69$.

In this research, CBCL was not used as a diagnostic instrument; rather it was used to gauge children's need for further

Table 1.

Descriptive data of CBCL for children under the age of 5.

\begin{tabular}{ccccc}
\hline & MIN & MAX & M & SD \\
\hline Emotionally Reactive & 50 & 83 & 62.94 & 9.74 \\
Anxious/Depressed & 50 & 87 & 62.28 & 8.02 \\
Somatic Complaints & 50 & 84 & 63.05 & 9.40 \\
Withdrawn & 50 & 100 & 75.46 & 13 \\
Attention Problem & 50 & 80 & 63.57 & 8.01 \\
Aggressive Behavior & 50 & 88 & 62.26 & 9.36 \\
Sleep Problems & 50 & 100 & 59.58 & 10.86 \\
Internalizing Problem & 41 & 85 & 67.88 & 9.38 \\
Externalizing Problem & 32 & 85 & 62.52 & 9.96 \\
Total Problem & 35 & 90 & 68.00 & 10.95 \\
\hline
\end{tabular}


Table 2.

Descriptive data of CBCL for children over 6 years old.

\begin{tabular}{ccccc}
\hline & MIN & MAX & M & SD \\
\hline Anxious/Depressed & 50 & 82 & 61.33 & 9.84 \\
Withdrawn/Depressed & 50 & 88 & 64.93 & 11.34 \\
Somatic Complaints & 50 & 74 & 58.96 & 7.35 \\
Rule-Breaking Behavior & 50 & 76 & 60.74 & 8.02 \\
Aggressive Behavior & 50 & 97 & 67.26 & 12.46 \\
Social Problems & 9 & 88 & 65.07 & 13.97 \\
Thought Problems & 50 & 84 & 66.11 & 9.38 \\
Attention Problems & 53 & 97 & 71.78 & 11.73 \\
Internalizing Problem & 39 & 81 & 62.96 & 11.19 \\
Externalizing Problem & 41 & 82 & 64.33 & 9.94 \\
Total Problem & 44 & 83 & 67.56 & 9.70 \\
\hline
\end{tabular}

clinical observation and assessment.

The parent-reported Vineland II gave a comprehensive overview about a child's developmental status in language, communication, living skills and motor skills. Table 3 showed that $14.6 \%$ of the parents estimated their children's receptive lan- guage ability to be very low, and $23.3 \%$ of the parents estimated their children's expressive language ability to be very low. They further rated their children's communication ability, relationship, and play and leisure time as relatively low.

Table 4 shows the distribution of the adaptive levels of the Mullen scores. The proportion of the adaptive levels of children's receptive and expressive language assessed by counselling psychologists (see Table 4) was congruent with the estimation of the parents (see Table 3). However, there were more children whose fine motor skills were assessed by counselling psychologists as very low as compared with the estimation by parents.

For children who were suspected to have autism disorder, as well as for those who did not present with obvious symptoms of autism but had been assessed by the Health Department state to have autism, the Childhood Autism Rating Scale (Schopler, Reichler, DeVellis, \& Daly, 1980) was administered.

In general, the assessment in this program served an informative rather than diagnostic function. The strengths and weaknesses in the child's developmental aspects were accentuated for the development of a continuous IDP, in lieu of categorizing

Table 3.

Frequency distribution of adaptive levels of the Vineland scores (Sparrow et al., 2005, 2008).

\begin{tabular}{|c|c|c|c|c|c|c|c|c|c|c|}
\hline & \multicolumn{2}{|c|}{ Very low } & \multicolumn{2}{|c|}{ Below average } & \multicolumn{2}{|c|}{ Average } & \multicolumn{2}{|c|}{ Above average } & \multicolumn{2}{|c|}{ Very high } \\
\hline & $f$ & $\%$ & $f$ & $\%$ & $f$ & $\%$ & $f$ & $\%$ & $f$ & $\%$ \\
\hline Receptive & 15 & 14.6 & 32 & 31.1 & 41 & 39.8 & 10 & 9.7 & 0 & 0 \\
\hline Expressive & 24 & 23.3 & 27 & 26.2 & 46 & 44.7 & 0 & 0 & 1 & 1.0 \\
\hline Written & 2 & 1.9 & 11 & 10.7 & 45 & 43.7 & 14 & 13.6 & 2 & 1.9 \\
\hline Communication & 14 & 13.6 & 30 & 29.1 & 52 & 50.5 & 2 & 1.9 & 0 & 0 \\
\hline Personal & 7 & 6.8 & 26 & 25.2 & 57 & 55.3 & 7 & 6.8 & 0 & 0 \\
\hline Domestic & 2 & 1.9 & 27 & 26.2 & 66 & 64.1 & 3 & 2.9 & 0 & 0 \\
\hline Community & 5 & 4.9 & 25 & 24.3 & 51 & 49.5 & 16 & 15.5 & 1 & 1.0 \\
\hline Living Skills & 3 & 2.9 & 28 & 27.2 & 62 & 60.2 & 5 & 4.9 & 0 & 0 \\
\hline Relationships & 17 & 16.5 & 41 & 39.8 & 40 & 38.8 & 0 & 0 & 0 & 0 \\
\hline Play and Leisure Time & 23 & 22.3 & 43 & 41.7 & 32 & 31.1 & 0 & 0 & 0 & 0 \\
\hline Coping Skills & 2 & 1.9 & 25 & 24.3 & 69 & 67.0 & 2 & 1.9 & 0 & 0 \\
\hline Socialization & 7 & 6.8 & 42 & 40.8 & 49 & 47.6 & 0 & 0 & 0 & 0 \\
\hline Gross Motor Skills & 8 & 7.8 & 34 & 33.0 & 54 & 52.4 & 2 & 1.9 & 0 & 0 \\
\hline Fine Motor Skills & 5 & 4.9 & 35 & 34.0 & 55 & 53.4 & 3 & 2.9 & 0 & 0 \\
\hline Motor Skills & 8 & 7.8 & 40 & 38.8 & 49 & 47.6 & 1 & 1.0 & 0 & 0 \\
\hline
\end{tabular}

Table 4.

Frequency distribution of adaptive levels of Mullen scores (Mullen, 1995).

\begin{tabular}{|c|c|c|c|c|c|c|c|c|c|c|}
\hline & \multicolumn{2}{|c|}{ Very low } & \multicolumn{2}{|c|}{ Below average } & \multicolumn{2}{|c|}{ Average } & \multicolumn{2}{|c|}{ Above average } & \multicolumn{2}{|c|}{ Very high } \\
\hline & $f$ & $\%$ & $f$ & $\%$ & $f$ & $\%$ & $f$ & $\%$ & $f$ & $\%$ \\
\hline Gross $^{*}$ Motor & 1 & 1.0 & 4 & 3.9 & 6 & 5.8 & 1 & 1.0 & 0 & 0 \\
\hline Fine Motor & 24 & 23.3 & 9 & 8.7 & 21 & 20.4 & 7 & 6.8 & 4 & 3.9 \\
\hline Receptive Language & 23 & 22.3 & 15 & 14.6 & 20 & 19.4 & 5 & 4.9 & 2 & 1.9 \\
\hline Early Learning Composite & 28 & 27.2 & 10 & 9.7 & 19 & 18.4 & 6 & 5.8 & 1 & 1.0 \\
\hline
\end{tabular}

Note: ${ }^{*}$ Gross motor assessment is solely for children under the age of 33 month and 30 days. 
children in pathological terms.

\section{Intervention: Individualized Development Plan (IDP)}

The intervention for children consisted of two parts: individual counselling/training and group training. The IDP summarized the child's developmental history, assessment results, and need for counselling and training. The IDP facilitated parents to keep track of the progress of their children throughout the twoyear program. Based on the assessment results, individualized goals aiming at helping children reach their developmental milestones were set in the three domains of communication, social skills and daily living skills.

The plan was explained to parents who were involved in the discussion of strategies to help children achieve the requisite training tasks. It was important to convey an attitude of positivism to parents to facilitate their maintenance of a sense of control. Children were then allocated to different streams based on their results in the Vineland and Mullen tests.

\section{Streamlining}

The scores on the Vineland and Mullen clearly indicated the domains where the children needed training. Children were allocated according to the Streaming Decision Matrix. For example, a child who scored two standard deviations below the mean in the communication domain was streamed into the "Severe Communication Training" group. Altogether, there were three domains of training: communication skills, social skills, and daily living skills. The streaming was conducted on the basis of functionality, hence diagnostic features were not emphasized. Hopefully, such a measure would minimize the effects of labeling, and shift parents' mindset from a problem orientation to that of a needs orientation.

\section{Group Training Aspects}

The group training in each of the three domains was divided into three levels of mild, moderate and severe. Each child was assigned to one of the four-session group training according to their streaming. The training tasks in each domain were designed to specifically strengthen the child's ability in that domain. For example, in the Communication Training Group, the training areas included receptive language, expressive language, comprehension of sentences and muscle training. The difficulties of the tasks were adjusted according to the child's streaming. For instance, in the Severe-Communication Group, children were required to practice on following one-part instruction; while in the Mild-Communication Group, children were required to follow two-part or more complicated instruction.

Children were encouraged to complete their tasks, and in so doing, it was hoped that their sense of self-efficacy could be enhanced.

\section{Individual Therapy and Training}

Children were also streamed to receive individual services in accordance with their presented abilities in the three domains. Overall, children with lower ability received more frequent intervention. In practice, children who scored two standard deviations below the mean on the Vineland and/or Mullen scales were categorized as severe cases; those who scored one standard deviation below were categorized as moderate cases; and other who obtained scores higher than the mean were categorized as mild cases. For severe cases, children received individual intervention every two weeks; moderate cases received individual intervention every four weeks; and mild cases received individual intervention every six weeks.

The individual intervention included training, therapy and parent consultation. The training areas also covered the three domains of communication skills, social skills and daily living skills. The tasks were tailored-made according to the goals set in the IDP and the daily habits of the children. The tasks vary from structured table task to non-structured free play. Word cards, color pen, dollhouse, storybooks and other different toys were used in the training and therapy with children. Parenting skills and training skills to practice at home were taught in parents' consultation session.

\section{Discussion: Functional Approach}

Functional approach is used consistently throughout the whole program.

First, contrary to the traditional medical model which focuses on diagnosing and medication, our program chose a functional approach in selecting measures to understand the children's psychological and behavioral status. The evaluation of each child was based on both the Children Behavior Checklist, and the Vineland Adaptive Behavior Scales-Second Edition. This provided a more comprehensive understanding of children's overall functioning, including their strengths and weaknesses in communication, social skills, daily living skills and motor skills. Based on this information, counselling psychologists were able to design a more appropriate IDP for the children, while simultaneously facilitating parents and other professionals to follow through the plan.

Second, the functional approach also suggests effective parent-psychologist collaboration. Under the time constraints of each training session, it was more beneficial to the children if parents were able to acquire home-based training techniques and apply them on their children. To facilitate this, it was important for psychologists to use layman terms to communicate with parents, instead of dumping diagnostic criteria, assessment data, and clinical terms on them.

Third, the children were streamed according to their age and developmental needs, while their diagnostic features were not emphasized. This minimized the labeling effect and shifted the parents from a problem-oriented mindset into a needs-oriented mindset. With the focus of the developmental needs of children, the trainings were designed to achieve developmental milestones instead of removing any symptoms. The difficulties of training tasks were set according to the ability of the children. Children were encouraged during the tasks and this allowed for the development of self-efficacy.

Fourth, the IDP itself also adopted a functional approach. It was tailor-made to fit the unique developmental needs of each child by suggesting the specific developmental tasks that needed to be mastered, and by fully engaging parents in achieving this mastery.

\section{Limitation}

Regardless of the theoretical and practical implications, limitations of the present program should be recognized. First, 
due to the lack of the Hong Kong norm of Vineland (Sparrow et al., 2005, 2008) and Mullen (Mullen, 1995), we had to use the Norm of the States as reference to estimate children's development status. Even if the assessment results showed consistency with our clinical observation, our experience suggested a need for future researches in exploring the Hong Kong norms of assessment tools for measuring cognitive and motor abilities of pre-school children. Second, the present program is still ongoing. Although our counselling psychologists have observed noticeable progress of most of the children, we still need to collect phase II and phase III data of this embedded longitudinal research with repeated measures before we can comprehensively evaluate the effect of the functional approach adopted in this program. Last but not least, the ideas of integrated case management, individualized developmental plan, and a functional approach are the ideas that we cherished in this program. We do realize that they are, at this stage, not well developed yet. However, we hope the sharing of what we currently have may generate more inspiring discussion.

\section{Conclusion}

Brain plasticity is a significant feature in early childhood. Thus, in cases of children with confirmed or suspected SEN, it is crucial that early diagnosis and early intervention are instituted. Unfortunately, this has not been possible in the Hong Kong context where the focus is placed on education, not development, and children with SEN do not receive resources for diagnosis and intervention until they enter the primary school system. This project is an attempt to demonstrate the benefits of early diagnosis and intervention by adopting a functional approach instead of the traditional diagnostic approach. Although this is an ongoing project and it is still premature to speak of definitive findings, yet the subjective feedback we have received from parents and caregivers have been positive. We are encouraged, and look forward to the development of a model of the functional approach in the early diagnosis and intervention with SEN children.

\section{Acknowledgements}

This early diagnosis and early intervention program of Hong Kong pre-school children with SEN is funded by Lok Sin Tong and the embedded research has obtained ethical approval from the Human Research Committee of Hong Kong Shue Yan University.

\section{REFERENCES}

Achenbach, T. (1991). Manual for the child behavior checklist/4-18 and 1991 profile. Burlington, VT: University of Vermont Department of Psychiatry.

Achenbach, T. (1992). Manual for the child behavior checklist/2-3 and 1992 profile. Burlington, VT: University of Vermont Department of Psychiatry.

Chlebowski, C., Green, J. A., Barton, M. L., \& Fein, D. (2010). Using the childhood autism rating scale to diagnose autism spectrum disorders. Journal of Autism \& Developmental Disorders, 40, 787-799. http://dx.doi.org/10.1007/s10803-009-0926-X

Chung, S. Y., Luk, S. L., \& Lee, P. W. H. (1990). A follow-up of infantile autism in Hong Kong. Journal of Autism and Developmental Disorder, 20, 221-232. http://dx.doi.org/10.1007/BF02284720

Hong Kong Education Bureau (2008). A tool for school self-evaluation and school development.

http://www.edb.gov.hk/attachment/en/edu-system/special/policy-and -initiatives/indicators-082008_e.pdf

Hong Kong Education Bureau (2013). Special education services. http:/www.edb.gov.hk/en/edu-system/special/overview/factsheet/spe cial-edu-serv/index.html

Lam, M.K., \& Rao, N. (1993). Developing a Chinese version of the psychoeducational profile (CPEP) to assess children in Hong Kong. Journal of Autism and Developmental Disorders, 23, 273-279. http://dx.doi.org/10.1007/BF01046220

Landa, R. J. (2008). Diagnosis of autism spectrum disorders in the first 3 years of life. Nature Clinical Practice Neurology, 4, 138-147. http://dx.doi.org/10.1038/ncpneuro0731

Leung, P. W. L., Lee, C., Hung, F. et al. (2005). Dopamine receptor D4 (DRD4) gene in Han Chinese children with attention-deficit/hyperactivity disorder (ADHD): Increased prevalence of the 2-repeat allele. Dopamine receptor D4 (DRD4) gene in Han Chinese children with attention-deficit/hyperactivity disorder (ADHD): Increased prevalence of the 2-repeat allele. American Journal of Medical Genetics Part B: Neuropsychiatric Genetics, 133, 54-56. http://dx.doi.org/10.1002/ajmg.b.30129

McBride-Chang, C. Lam, F., Lam, C., Doo, S., Wong, S. W. L., \& Chow, Y. (2008). Word recognition and cognitive profiles of Chinese pre-school children at risk for dyslexia through language delay or familial history of dyslexia. Journal of Child Psychology and Psychiatry, 49, 211-218.

McBride-Chang, C., Shu, H., Zhou, A., Wat, C. P., \& Wagner, R. K. (2003). Morphological awareness uniquely predicts young children's Chinese character recognition. Journal of Educational Psychology, 95, 743-751. http://dx.doi.org/10.1037/0022-0663.95.4.743

Mullen, E. M. (1995). Mullen scales of early learning (AGS ed.). Circle Pines, MN: American Guidance Service Inc.

Rappaport, G. C., Ornoy, A., \& Tenenbaum, A. (1998). Is early intervention effective in preventing ADHD? The Israel Journal of Psychiatry and Related Sciences, 35, 271-279.

Schopler, E., Reichler, R. J., DeVellis, R. F., \& Daly, K. (1980). Toward objective classification of childhood autism: Childhood Autism Rating Scale (CARS). J Autism DevDisord, 10, 91-103. http://dx.doi.org/10.1007/BF02408436

Sparrow, S. S., Cicchetti, D. V., \& Balla, D. A. (2005). Vineland adaptive behavior scales (2nd ed.). Circle Pines, MN: American Guidance Service.

Sparrow, S. S., Cicchetti, D. V., \& Balla, D. A. (2008). Vineland adaptive behavior scales: Second edition (vineland II), the expanded interview form. Livonia, MN: Pearson Assessments.

Victoria, Z., Areti, A., Pavls, C., Lambros, S., Ioanna, S., Venetsanos, M., \& Meropi, T. (2011). An interpretative model of early indicators of specific developmental dyslexia in preschool age: A comparative presentation of three studies in Greece. Research in Developmental Disabilities, 32, 3003-3016. http://dx.doi.org/10.1016/j.ridd.2011.03.021

Yuen, M., \& Westwood, P. (2001). Integratiing students with special nees in Hong Kong secondary schools: Teachers' attitudes and their possible relationship to guidance training. International Journal of Special Education, 16, 69-84. 